Article

\title{
Four New Glycosides from the Fruit of Xanthium sibiricum Patr.
}

\author{
Hai Jiang, Liu Yang, Chang Liu, Hui Hou, Qiuhong Wang, Zhibin Wang, Bingyou Yang \\ and Haixue Kuang *
}

Key Laboratory of Chinese Materia Medica, Ministry of Education, Heilongjiang University of Chinese Medicine, Harbin 150040, China

* Author to whom correspondence should be addressed; E-Mail: hxkuang56@163.com; Tel.: +86-451-8219-3001; Fax: +86-451-8211-0803.

Received: 19 July 2013; in revised form: 26 September 2013 / Accepted: 29 September 2013 / Published: 10 October 2013

\begin{abstract}
Four new glycosides, namely $3 \beta$-norpinan-2-one 3- $O$ - $\beta$-D-apiofuranosyl- $(1 \rightarrow 6)$ $\beta$-D-glucopyranoside (1), (6Z)-3-hydroxymethyl-7-methylocta-1,6-dien-3-ol 8-O- $\beta$-Dglucopyranoside (2), (6E)-3-hydroxymethyl-7-methylocta-1,6-dien-3-ol 8-O- $\beta$-D-glucopyranoside $(3)$, and 7-[( $\beta$-D-apiofuranosyl- $(1 \rightarrow 6)-\beta$-D-glucopyranosyl)oxymethy $]-8,8$ dimethyl-4,8-dihydrobenzo[1,4]thiazine-3,5-dione (4), were isolated from the fruits of Xanthium sibiricum Patr together with three known compounds, xanthiside (5), adenosine (6), and 2,3-dihydroxy-1-(4-hydroxy-3-methoxyphenyl)-propan-1-one (7). The structures of the new compounds were determined on the basis of detailed spectroscopic analyses.
\end{abstract}

Keywords: Xanthium sibiricum Patr.; fruits; glycosides

\section{Introduction}

The fruits of Xanthium sibiricum Patr. (Compositae), hereafter defined as "Fructus Xanthii", known as Cang er zi in Traditional Chinese Medicine, are used for treating nasal sinusitis, numbness of limbs, arthritis, ulcer, pruritus, cancer, and herpes [1-6]. Many chemical studies on Fructus Xanthii have been conducted and several essential oils, amino acids, organic acids, sesquiterpene lactones, diterpenes, and thiazinediones have been isolated [7-17]. Moreover, the $n$-BuOH fraction of Fructus Xanthii has been shown to possess anti-inflammatory properties [18], and has been used for controlling macrophage-mediated inflammatory diseases [19]. However, there are few reports on the screening of bioactive components of $X$. sibiricum. Our study, which focused on the evaluation of anti-inflammatory 
properties of Fructus Xanthii, aimed to determine the most active constituents of the plant. Herein, we present the isolation and structure elucidation of four new compounds: $3 \beta$-norpinan-2-one 3-O- $\beta$-Dapiofuranosyl-( $1 \rightarrow 6)$ - $\beta$-D-glucopyranoside (1), (6Z)-3-hydroxymethyl-7-methylocta-1,6-dien-3-ol 8$O$ - $\beta$-D-glucopyranoside (2), (6E)-3-hydroxymethyl-7-methylocta-1,6-dien-3-ol $\quad 8$ - $O$ - $\beta$-D-glucopyranoside $(3)$, and 7-[( $\beta$-D-apiofuranosyl-( $1 \rightarrow 6)$ - $\beta$-D-glucopyranosyl)oxymethy]-8,8-dimethyl-4,8dihydrobenzo[1,4]thiazine-3,5-dione (4), as well as three known compounds: xanthiside (5) [14], adenosine (6) [20], and 2,3-dihydroxy-1-(4-hydroxy-3-methoxyphenyl)-propan-1-one (7) [21], from the ethanol extract of Fructus Xanthii (Figure 1).

Figure 1. Structures of compounds 1-7 isolated from Fructus Xanthii.

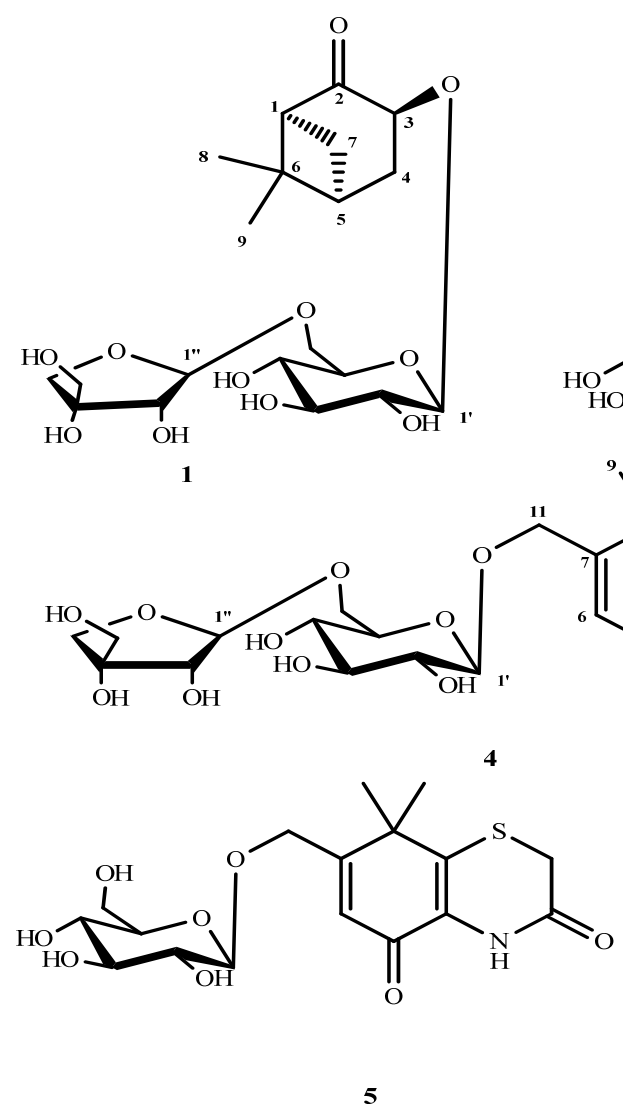

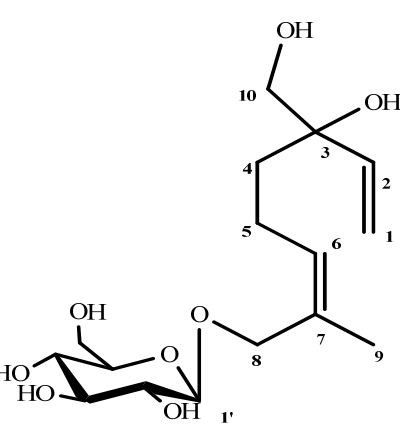

2<smiles>CC(C)(C)C1=C(C(C)(C)C)NC(=O)CSC1</smiles><smiles>Nc1ncc2ncn(C3OC(O)C(O)C(O)C3O)c2n1</smiles>

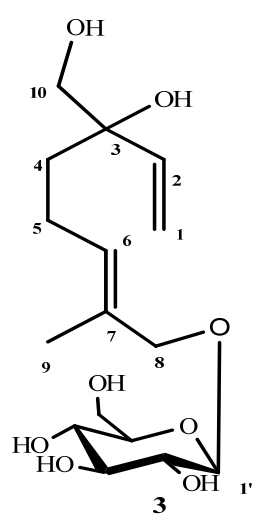<smiles>COc1cc(C(=O)C(O)CO)ccc1O</smiles>

\section{Results and Discussion}

Compound 1 was obtained as a white amorphous powder with molecular formula $\mathrm{C}_{20} \mathrm{H}_{32} \mathrm{O}_{11}$ (deduced from the HRESIMS and NMR data). The ${ }^{1} \mathrm{H}-\mathrm{NMR}$ spectrum showed proton signals for two methyl groups at $\delta 0.79(3 \mathrm{H}, \mathrm{s}, \mathrm{Me}-8)$ and $1.36(3 \mathrm{H}, \mathrm{s}, \mathrm{Me}-9)$, and two anomeric proton signals of sugar moieties at $\delta 4.50\left(1 \mathrm{H}, \mathrm{d}, J=7.8 \mathrm{~Hz}, \mathrm{H}-1^{\prime}\right)$ and $5.01\left(1 \mathrm{H}, \mathrm{d}, J=2.5 \mathrm{~Hz}, \mathrm{H}-1^{\prime}\right)$. The ${ }^{13} \mathrm{C}$-NMR spectrum of $\mathbf{1}$ displays 20 carbon signals separated by DEPT experiments into two methyl, five methylene, 10 methine, and three quaternary carbon moieties. The structure of $\mathbf{1}$ was also confirmed by $2 \mathrm{D}$ NMR experiments (Figure 2). In the ${ }^{1} \mathrm{H}-{ }^{1} \mathrm{H}$ COSY spectrum of $\mathbf{1}$, correlations were 
observed from $\mathrm{H}-1$ to $\mathrm{H}-7, \mathrm{H}-7$ to $\mathrm{H}-5, \mathrm{H}-5$ to $\mathrm{H}_{2}-4$, and $\mathrm{H}_{2}-4$ to $\mathrm{H}-3$. In the HMBC spectrum, some key correlations were observed from $\mathrm{H}-1$ to $\mathrm{C}-2, \mathrm{C}-3, \mathrm{C}-5, \mathrm{C}-6$ and $\mathrm{C}-7$; $\mathrm{H}-3$ to $\mathrm{C}-2$ and $\mathrm{C}-1$ '; $\mathrm{H}_{2}-4$ to C-2, C-3, C-5, and C-6; H-5 to C-1; $\mathrm{H}_{2}-7$ to C-1, C-2, C-4, and C-5; $\mathrm{H}_{3}-8$ to C-1, C-5, and C-9; $\mathrm{H}_{3}-9$ to $\mathrm{C}-1, \mathrm{C}-5$, and $\mathrm{C}-8$; H-1' to $\mathrm{C}-3$; and $\mathrm{H}-1$ ' to C-6'. From the analysis of the ${ }^{1} \mathrm{H}-{ }^{1} \mathrm{H}$ COSY and HMBC spectral data, the planar structure of compound 1 was determined. Therefore, the aglycone of 1 was concluded to be 3-hydroxynorpinan-2-one [22], and the location of its glycosyl group was found to be C-3. The NOESY correlations between $\mathrm{H}_{3}-8$ and $\mathrm{H}_{\beta}-4, \mathrm{H}_{3}-9$ and $\mathrm{H}_{\beta}-7, \mathrm{H}_{\alpha}-4$ and $\mathrm{H}_{\alpha}-7, \mathrm{H}_{\alpha^{-}}-4$ and $\mathrm{H}-3$, and between $\mathrm{H}_{\alpha}-7$ and $\mathrm{H}-3$ established the connective sites, as shown in the structure of 1 (Figure 3). Therefore, the relative configuration of $\mathrm{H}-3$ is $\alpha$. Acid hydrolysis of 1 liberated D-glucose and D-apiose, which were identified by HPLC with optical rotation detection [23,24]. By comparing the coupling constants and chemical shifts of sugar signals with those of the reported sugars $[25,26]$, the two sugars were deduced to be the $\beta$-configuration of glucose and $\beta$ for apiose. From these results, 1 was determined to be $3 \beta$-norpinan-2-one 3-O- $\beta$-D-apiofuranosyl- $(1 \rightarrow 6)-\beta$-D-glucopyranoside.

Figure 2. Key ${ }^{1} \mathrm{H}-{ }^{1} \mathrm{H}$ COSY and HMBC correlations of compounds $\mathbf{1}-\mathbf{4}$.
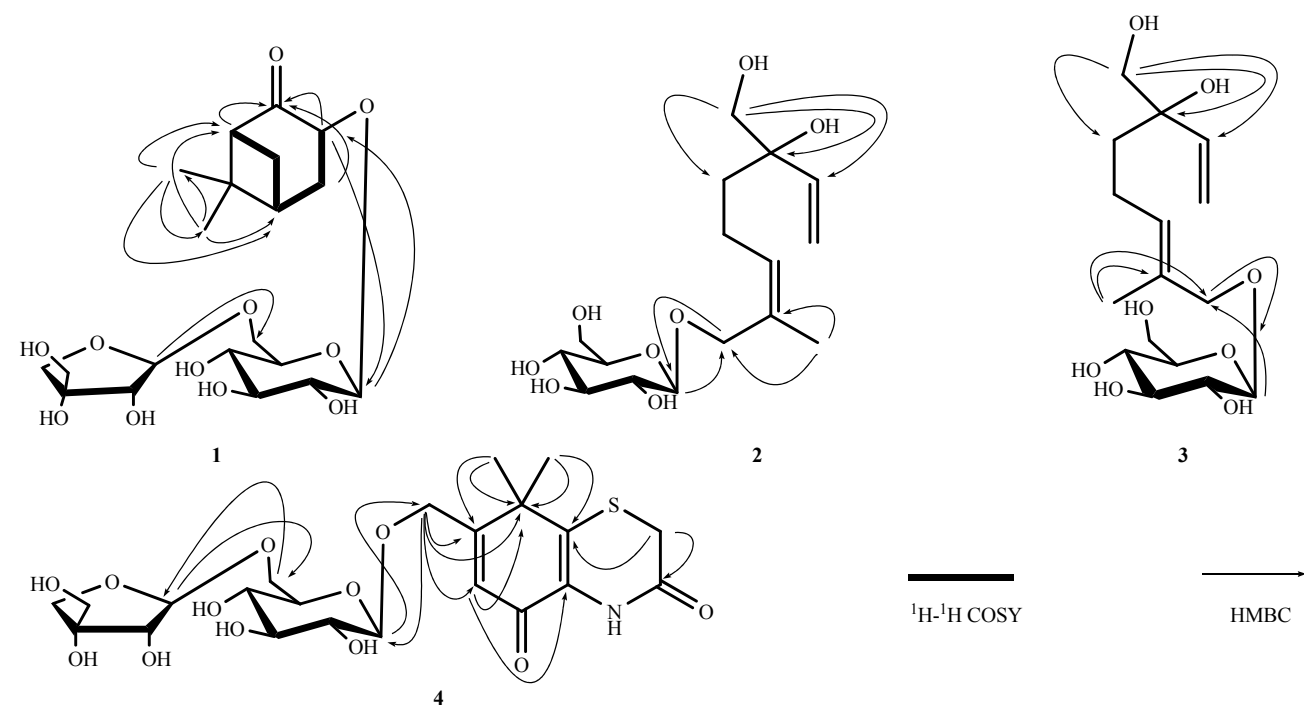

Compound 2 was obtained as a yellow amorphous powder with molecular formula $\mathrm{C}_{16} \mathrm{H}_{28} \mathrm{O}_{8}$ (determined by the HRESIMS and NMR data). Acid hydrolysis of 2 liberated D-glucose, which was identified by HPLC with optical rotation detection [24]. The ${ }^{1} \mathrm{H}-\mathrm{NMR}$ spectrum indicates the presence of a vinyl group, $\delta 5.15(1 \mathrm{H}, \mathrm{dd}, J=1.7,10.9 \mathrm{~Hz}, \mathrm{H}-1 \mathrm{a}), 5.27(1 \mathrm{H}, \mathrm{dd}, J=1.7,17.4 \mathrm{~Hz}, \mathrm{H}-1 \mathrm{~b})$, and $5.89(1 \mathrm{H}, \mathrm{dd}, J=10.9,17.4 \mathrm{~Hz}, \mathrm{H}-2)$; an additional olefinic hydrogen, $\delta 5.41(1 \mathrm{H}$, brt, $J=7.0 \mathrm{~Hz}, \mathrm{H}-6)$; four methylene protons, $\delta 1.54(1 \mathrm{H}, \mathrm{ddd}, J=9.6,6.0,2.4 \mathrm{~Hz}, \mathrm{H}-4 \mathrm{a}), 1.57(1 \mathrm{H}$, ddd, $J=9.6,6.0,2.4 \mathrm{~Hz}, \mathrm{H}-4 \mathrm{~b}), 2.05(1 \mathrm{H}, \mathrm{m}, \mathrm{H}-5 \mathrm{a})$, and 2.15 (1H, m, H-5b); two oxygenated methylene groups, $\delta 3.40\left(2 \mathrm{H}, \mathrm{d}, J=2.6 \mathrm{~Hz}, \mathrm{H}_{2}-10\right), 4.35(1 \mathrm{H}, \mathrm{d}, J=11.4 \mathrm{~Hz}, \mathrm{H}-8 \mathrm{a})$, and $4.20(1 \mathrm{H}, \mathrm{d}$, $J=11.4 \mathrm{~Hz}, \mathrm{H}-8 \mathrm{~b})$; one methyl group, $\delta 1.76(3 \mathrm{H}, \mathrm{s}, \mathrm{H}-9)$; and an anomeric proton of a gluco-pyranoside, $\delta 4.21\left(1 \mathrm{H}, \mathrm{d}, J=7.8 \mathrm{~Hz} \mathrm{H}-1^{\prime}\right)$. From the coupling constant of the anomeric proton, $\mathrm{C}-1$ ' of D-glucose was determined to be in the $\beta$-configuration. In the ${ }^{13} \mathrm{C}$-NMR and DEPT spectra, 16 signals except six signals resulting from the glucopyranosyl moiety were observed. These peaks suggest the presence of an aliphatic monoterpene skeleton. Thus, signals for two sets of double bonds 


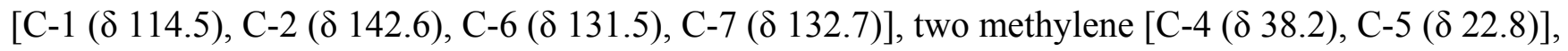
two oxygenated methylenes $[\mathrm{C}-8(\delta$ 67.8), C-10 ( $\delta$ 69.5)], one methyl [C-9 $(\delta$ 21.9)] moieties were observed, together with a quaternary carbon signal at $\delta 76.7(\mathrm{C}-3)$. In the HMBC spectrum, the key correlations were observed from $\mathrm{H}_{3}-9$ to $\mathrm{C}-7$ and $\mathrm{C}-8 ; \mathrm{H}_{2}-10$ to $\mathrm{C}-2, \mathrm{C}-3$, and $\mathrm{C}-4$; and $\mathrm{H}-1$ ' to C-8. Therefore, the glycosyl group is located at C-8. A diagnostic cross peak was observed between the methyl hydrogen $\left(\mathrm{H}_{3}-9\right)$ and the olefinic hydrogen (H-6) in the NOESY spectrum (Figure 3 ). Thus, the stereochemistry of the double-bond system between C-6 and C-7 in 2 was confirmed to have the $Z$ orientation. Even though the configuration of C-3 of 2 was not defined, this is the first report of the isolation of an acyclic monoterpene glycoside from Fructus Xanthii. Therefore, compound 2 was determined to be (6Z)-3-hydroxymethyl-7-methylocta-1,6-dien-3-ol 8-O- $\beta$-D-glucopyranoside.

Figure 3. Main NOE correlations of compounds 1-3.
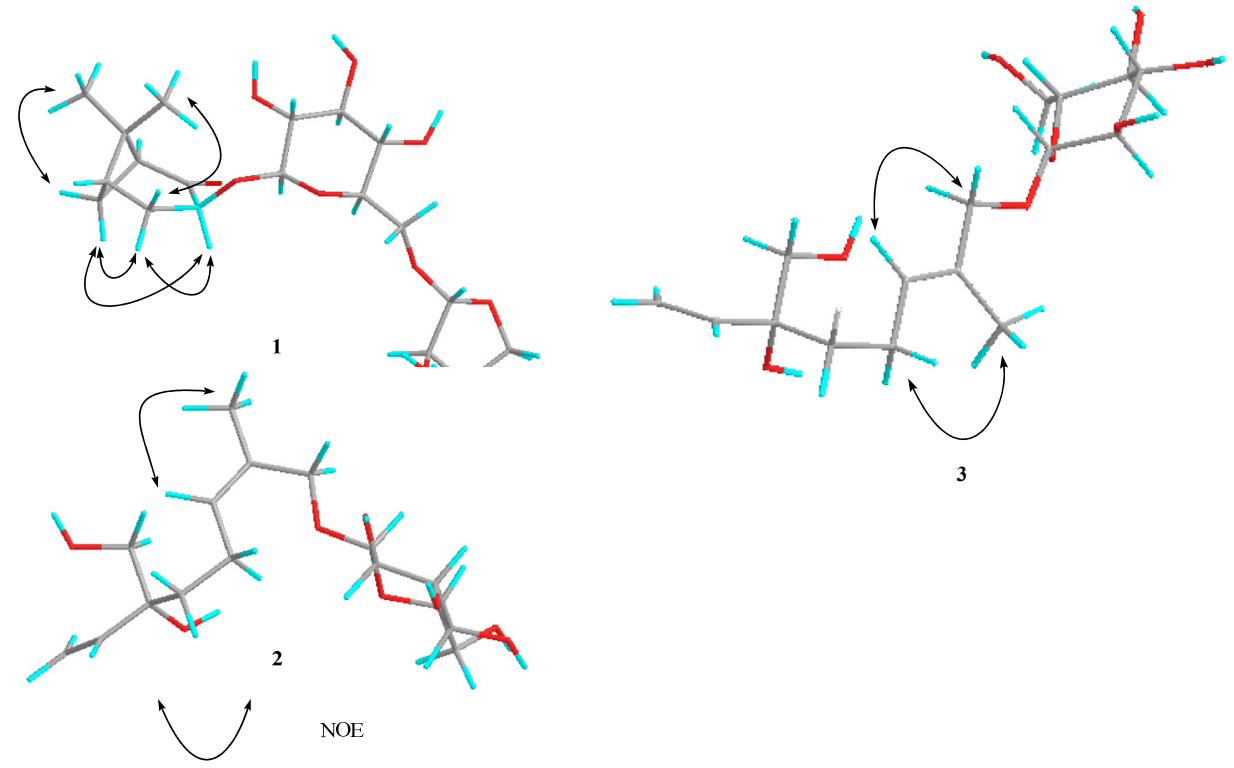

Compound $\mathbf{3}$ was also obtained as a yellow amorphous powder. The positive-ion HRESIMS of $\mathbf{3}$ exhibits a quasi-molecular ion peak at $m / z 371.1685[\mathrm{M}+\mathrm{Na}]^{+}$. The molecular formula of $\mathbf{3}\left(\mathrm{C}_{16} \mathrm{H}_{28} \mathrm{O}_{8}\right)$ was determined from the quasi-molecular ion peak and HRESIMS measurements. Acid hydrolysis of $\mathbf{3}$ liberated D-glucose, which was identified by HPLC with optical rotation detection [24]. ${ }^{1} \mathrm{H}-\mathrm{NMR}$ $\left(\mathrm{CD}_{3} \mathrm{OD}\right)$ and ${ }^{13} \mathrm{C}$-NMR spectra (Tables 1 and 2 ) of $\mathbf{3}$, which were examined using the results of various NMR experiments, show signals assignable to an aglycone moiety $[\delta 5.30(1 \mathrm{H}, \mathrm{dd}, J=1.6$, $17.4 \mathrm{~Hz}, \mathrm{H}-1 \mathrm{a}), 5.17(1 \mathrm{H}, \mathrm{dd}, J=1.6,10.9 \mathrm{~Hz}, \mathrm{H}-1 \mathrm{~b})],[\delta 5.88(1 \mathrm{H}, \mathrm{dd}, J=17.4,10.9 \mathrm{~Hz}, \mathrm{H}-2)]$,

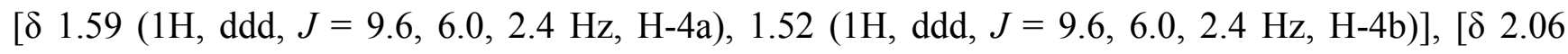
$(1 \mathrm{H}, \mathrm{m}, \mathrm{H}-5 \mathrm{a}), 2.16(1 \mathrm{H}, \mathrm{m}, \mathrm{H}-5 \mathrm{~b})],[\delta 5.48(1 \mathrm{H}, \mathrm{brt}, J=6.5 \mathrm{~Hz}, \mathrm{H}-6)],[\delta 4.18(1 \mathrm{H}, \mathrm{d}, J=11.5 \mathrm{~Hz}$, $\mathrm{H}-8 \mathrm{a}), 4.03(1 \mathrm{H}, \mathrm{d}, J=11.5 \mathrm{~Hz}, \mathrm{H}-8 \mathrm{~b})],[\delta 1.68(3 \mathrm{H}, \mathrm{s}), \mathrm{H}-9],[\delta 3.41(2 \mathrm{H}, \mathrm{d}, J=3.2 \mathrm{~Hz})]$ and a $\beta$-D-glucopyranosyl moiety [ $\delta 4.23(1 \mathrm{H}, \mathrm{d}, J=7.8 \mathrm{~Hz})]$. All ${ }^{1} \mathrm{H}-\mathrm{NMR}$ and ${ }^{13} \mathrm{C}$-NMR data were similar to those for 2. However, an important difference observed for this part was the chemical shift value of the vinyl methyl carbon, $\delta 14.1$ (C-9). Analysis of the ${ }^{13} \mathrm{C}-\mathrm{NMR}$ spectra revealed signal values similar to those reported for creoside II [27]; thus, the double bond of $\mathbf{3}$ was determined to be trans. In addition, the geometry of $\mathbf{3}$ was also confirmed on the basis of NOESY results, which showed NOE 
correlations between the following proton pairs: $\mathrm{H}_{2}-5$ and $\mathrm{H}_{3}-9$; $\mathrm{H}-6$ and $\mathrm{H}_{2}-8$ (Figure 3). Thus, the stereochemistry of the double bond system between C-6 and C-7 in 3 was confirmed to be of the $E$ form. Thus, compound 3 was determined to be (6E)-3-hydroxymethyl-7-methylocta-1,6-dien-3-ol 8-O- $\beta$-D-glucopyranoside.

Table 1. ${ }^{1} \mathrm{H}-\mathrm{NMR}$ data for compounds $\mathbf{1}-\mathbf{4}\left(400 \mathrm{MHz}\right.$ in $\left.\mathrm{CD}_{3} \mathrm{OD}\right)$.

\begin{tabular}{|c|c|c|c|c|}
\hline No. & 1 & 2 & 3 & 4 \\
\hline \multirow{2}{*}{1} & \multirow{2}{*}{$2.58(1 \mathrm{H}, \mathrm{t}, J=5.4 \mathrm{~Hz})$} & $5.27(1 \mathrm{H}, \mathrm{dd}, J=1.7,17.4 \mathrm{~Hz})$ & $5.30(1 \mathrm{H}, \mathrm{dd}, J=1.6,17.4 \mathrm{~Hz})$ & \\
\hline & & $5.15(1 \mathrm{H}, \mathrm{dd}, J=1.7,10.9 \mathrm{~Hz})$ & $5.17(1 \mathrm{H}, \mathrm{dd}, J=1.6,10.9 \mathrm{~Hz})$ & \\
\hline 2 & & $5.89(1 \mathrm{H}, \mathrm{dd}, J=17.4,10.9 \mathrm{~Hz})$ & $5.88(1 \mathrm{H}, \mathrm{dd}, J=17.4,10.9 \mathrm{~Hz})$ & $3.48(2 \mathrm{H}, \mathrm{brs})$ \\
\hline 3 & $4.70(1 \mathrm{H}, \mathrm{dd}, J=6.9,10.6 \mathrm{~Hz})$ & & & \\
\hline \multirow{2}{*}{4} & $1.90(1 \mathrm{H}, \mathrm{dd}, J=6.9,13.6 \mathrm{~Hz})$ & $1.57(1 \mathrm{H}, \mathrm{ddd}, J=9.6,6.0,2.4 \mathrm{~Hz})$ & $1.59(1 \mathrm{H}, \mathrm{ddd}, J=9.6,6.0,2.4 \mathrm{~Hz})$ & \\
\hline & $2.77(1 \mathrm{H}, \mathrm{ddd}, J=4.6,10.6,13.6 \mathrm{~Hz})$ & $1.54(1 \mathrm{H}, \mathrm{ddd}, J=9.6,6.0,2.4 \mathrm{~Hz})$ & $1.52(1 \mathrm{H}, \mathrm{ddd}, J=9.6,6.0,2.4 \mathrm{~Hz})$ & \\
\hline \multirow{2}{*}{5} & \multirow{2}{*}{$2.22(1 \mathrm{H}, \mathrm{m})$} & $2.05(1 \mathrm{H}, \mathrm{m})$ & $2.06(1 \mathrm{H}, \mathrm{m})$ & \\
\hline & & $2.15(1 \mathrm{H}, \mathrm{m})$ & $2.16(1 \mathrm{H}, \mathrm{m})$ & \\
\hline 6 & & $5.41(1 \mathrm{H}$, brt, $J=7.0 \mathrm{~Hz})$ & $5.48(1 \mathrm{H}$, brt, $J=6.5 \mathrm{~Hz})$ & $6.67(1 \mathrm{H}, \mathrm{brs})$ \\
\hline \multirow{2}{*}{7} & $2.48(1 \mathrm{H}, \mathrm{m})$ & & & \\
\hline & $1.81(1 \mathrm{H}, \mathrm{m})$ & & & \\
\hline \multirow{2}{*}{8} & \multirow{2}{*}{$0.79(3 \mathrm{H}, \mathrm{s})$} & $4.35(1 \mathrm{H}, \mathrm{d}, J=11.4 \mathrm{~Hz})$ & $4.18(1 \mathrm{H}, \mathrm{d}, J=11.5 \mathrm{~Hz})$ & \\
\hline & & $4.20(1 \mathrm{H}, \mathrm{d}, J=11.4 \mathrm{~Hz})$ & $4.03(1 \mathrm{H}, \mathrm{d}, J=11.5 \mathrm{~Hz})$ & \\
\hline 9 & $1.36(3 \mathrm{H}, \mathrm{s})$ & $1.76(3 \mathrm{H}, \mathrm{s})$ & $1.68(3 \mathrm{H}, \mathrm{s})$ & $1.47(1 \mathrm{H}, \mathrm{s})$ \\
\hline 10 & & $3.40(2 \mathrm{H}, \mathrm{d}, J=2.6 \mathrm{~Hz})$ & $3.41(2 \mathrm{H}, \mathrm{d}, J=3.2 \mathrm{~Hz})$ & $1.47(1 \mathrm{H}, \mathrm{s})$ \\
\hline \multirow{2}{*}{11} & & & & $4.70(1 \mathrm{H}, \mathrm{d}, J=15.8 \mathrm{~Hz})$ \\
\hline & & & & $4.51(1 \mathrm{H}, \mathrm{d}, J=15.8 \mathrm{~Hz})$ \\
\hline Glc-1' & $4.50(1 \mathrm{H}, \mathrm{d}, J=7.8 \mathrm{~Hz})$ & $4.21(1 \mathrm{H}, \mathrm{d}, J=7.8 \mathrm{~Hz})$ & $4.23(1 \mathrm{H}, \mathrm{d}, J=7.8 \mathrm{~Hz})$ & $4.35(1 \mathrm{H}, \mathrm{d}, J=7.7 \mathrm{~Hz})$ \\
\hline $2^{\prime}$ & $3.24(1 \mathrm{H}, \mathrm{m})$ & $3.15(1 \mathrm{H}, \mathrm{dd}, J=8.9,7.8 \mathrm{~Hz})$ & $3.18(1 \mathrm{H}, \mathrm{m})$ & $3.30(1 \mathrm{H}, \mathrm{m})$ \\
\hline $3^{\prime}$ & $3.35(1 \mathrm{H}, \mathrm{m})$ & $3.25(1 \mathrm{H}, \mathrm{m})$ & $3.25(1 \mathrm{H}, \mathrm{m})$ & $3.34(1 \mathrm{H}, \mathrm{m})$ \\
\hline $4^{\prime}$ & $3.28(1 \mathrm{H}, \mathrm{m})$ & $3.30(1 \mathrm{H}, \mathrm{m})$ & $3.31(1 \mathrm{H}, \mathrm{m})$ & $3.25(1 \mathrm{H}, \mathrm{m})$ \\
\hline $5^{\prime}$ & $3.40(1 \mathrm{H}, \mathrm{m})$ & $3.35(1 \mathrm{H}, \mathrm{m})$ & $3.34(1 \mathrm{H}, \mathrm{m})$ & $3.41(1 \mathrm{H}, \mathrm{m})$ \\
\hline \multirow{2}{*}{$6^{\prime}$} & $3.98(1 \mathrm{H}, \mathrm{dd}, J=12.5,2.0 \mathrm{~Hz})$ & $3.87(1 \mathrm{H}, \mathrm{dd}, J=12.5,2.0 \mathrm{~Hz})$ & $3.85(1 \mathrm{H}, \mathrm{dd}, J=12.0,2.2 \mathrm{~Hz})$ & $3.98(1 \mathrm{H}, \mathrm{dd}, J=12.9,1.6 \mathrm{~Hz})$ \\
\hline & $3.60(1 \mathrm{H}, \mathrm{dd}, J=12.5,5.0 \mathrm{~Hz})$ & $3.67(1 \mathrm{H}, \mathrm{dd}, J=12.5,5.0 \mathrm{~Hz})$ & $3.65(1 \mathrm{H}, \mathrm{dd}, J=12.0,5.6 \mathrm{~Hz})$ & $3.61(1 \mathrm{H}, \mathrm{dd}, J=11.4,6.5 \mathrm{~Hz})$ \\
\hline Api-1" & $5.01(1 \mathrm{H}, \mathrm{d}, J=2.5 \mathrm{~Hz})$ & & & $5.01(1 \mathrm{H}, \mathrm{d}, J=2.4 \mathrm{~Hz})$ \\
\hline $2 "$ & $3.89(1 \mathrm{H}, \mathrm{d}, J=2.5 \mathrm{~Hz})$ & & & $3.89(1 \mathrm{H}, \mathrm{d}, J=2.4 \mathrm{~Hz})$ \\
\hline \multicolumn{5}{|l|}{$3 "$} \\
\hline \multirow{2}{*}{$4 "$} & $3.74(1 \mathrm{H}, \mathrm{d}, J=9.6 \mathrm{~Hz})$ & & & $3.95(1 \mathrm{H}, \mathrm{d}, J=9.6 \mathrm{~Hz})$ \\
\hline & $3.97(1 \mathrm{H}, \mathrm{d}, J=9.6 \mathrm{~Hz})$ & & & $3.74(1 \mathrm{H}, \mathrm{d}, J=9.6 \mathrm{~Hz})$ \\
\hline $5 "$ & $3.56(2 \mathrm{H}, \mathrm{s})$ & & & $3.48(2 \mathrm{H}, \mathrm{s})$ \\
\hline
\end{tabular}


Table 2. ${ }^{13} \mathrm{C}-\mathrm{NMR}$ data for compounds $\mathbf{1}-\mathbf{4}\left(100 \mathrm{MHz}\right.$ in $\left.\mathrm{CD}_{3} \mathrm{OD}\right)$.

\begin{tabular}{|c|c|c|c|c|}
\hline No. & 1 & 2 & 3 & 4 \\
\hline 1 & 57.9 & 114.5 & 114.5 & \\
\hline 2 & 215.2 & 142.6 & 142.5 & 29.8 \\
\hline 3 & 78.5 & 76.7 & 76.7 & 164.7 \\
\hline 4 & 32.9 & 38.2 & 37.5 & \\
\hline $4 a$ & & & & 131.0 \\
\hline 5 & 41.4 & 22.8 & 22.8 & 177.1 \\
\hline 6 & 45.5 & 131.5 & 130.3 & 123.4 \\
\hline 7 & 24.9 & 132.7 & 132.9 & 167.2 \\
\hline 8 & 21.9 & 67.8 & 75.9 & 43.5 \\
\hline $8 a$ & & & & 143.5 \\
\hline 9 & 26.3 & 21.9 & 14.1 & 27.5 \\
\hline 10 & & 69.5 & 69.7 & 27.4 \\
\hline 11 & & & & 67.9 \\
\hline Glc-1' & 105.9 & 102.4 & 102.6 & 103.9 \\
\hline $2^{\prime}$ & 75.5 & 75.0 & 75.1 & 75.1 \\
\hline $3^{\prime}$ & 77.9 & 77.9 & 77.9 & 78.0 \\
\hline $4^{\prime}$ & 71.6 & 71.7 & 71.7 & 71.7 \\
\hline $5^{\prime}$ & 77.1 & 78.2 & 78.2 & 77.2 \\
\hline $6^{\prime}$ & 68.7 & 62.8 & 62.8 & 68.7 \\
\hline Api-1" & 111.0 & & & 111.0 \\
\hline $2^{\prime \prime}$ & 78.0 & & & 78.0 \\
\hline 3" & 80.5 & & & 80.5 \\
\hline 4" & 75.0 & & & 75.0 \\
\hline $5^{\prime \prime}$ & 65.5 & & & 65.5 \\
\hline
\end{tabular}

Compound 4 was obtained as colorless needle-like crystals with molecular formula $\mathrm{C}_{22} \mathrm{H}_{31} \mathrm{NO}_{12} \mathrm{~S}$ (deduced from the HRESIMS and NMR data). The ${ }^{1} \mathrm{H}-\mathrm{NMR}$ spectrum of 4 reveals signals for an olefinic proton, $\delta 6.67\left(1 \mathrm{H}\right.$, brs, H-6); two methyls of a gem-dimethyl group, $\delta 1.47\left(6 \mathrm{H}, \mathrm{s}, \mathrm{H}_{3}-9,10\right)$; two oxygenated methylene protons, $\delta 4.70 / 4.51$ (each $1 \mathrm{H}, \mathrm{d}, J=15.8 \mathrm{~Hz}, \mathrm{H}-11 \mathrm{a} / \mathrm{H}-11 \mathrm{~b}$ ) and $\delta 3.48(2 \mathrm{H}$, brs, $\mathrm{H}-2)$; and two anomeric proton signals of sugar moieties at $\delta 4.35(1 \mathrm{H}, \mathrm{d}, J=7.7 \mathrm{~Hz}$, $\left.\mathrm{H}-1^{\prime}\right)$ and $5.01\left(1 \mathrm{H}, \mathrm{d}, J=2.4 \mathrm{~Hz}, \mathrm{H}-1^{\prime}\right)$. The ${ }^{13} \mathrm{C}-\mathrm{NMR}$ spectrum displays 22 carbon signals. Based on the DEPT and HSQC analyses, these signals were assigned to two double bonds at $\delta 123.4$ (C-6), 167.2 (C-7), 131.0 (C-4a), and 143.5 (C-8a); an oxygenated methylene, $\delta 67.9$ (C-11); an aliphatic methylene, $\delta 29.8$ (C-2); two methyls of a gem-dimethyl group, $\delta 27.5$ (C-9) and $27.4(\mathrm{C}-10)$; an aliphatic quaternary carbon, $\delta 43.5$ (C-8); and two quaternary carbons at $\delta 164.7$ (C-3) and 177.1 (C-5). Comparison of these data with those of thiazinedione, which was previously isolated from Xanthium strumarium [5], indicated that we are dealing with a glycoside derivative of thiazinedione. In the HMBC spectrum (Figure 2), the key correlations were observed from $\mathrm{H}_{3}-9 / 10$ to C-8, C-7, and C-8a; $\mathrm{H}_{2}-11$ to $\mathrm{C}-8, \mathrm{C}-7$, and $\mathrm{C}-6$; $\mathrm{H}-6$ to $\mathrm{C}-8, \mathrm{C}-4 \mathrm{a}$, and $\mathrm{C}-11$; and $\mathrm{H}_{2}-2$ to $\mathrm{C}-8 \mathrm{a}$ and $\mathrm{C}-3$. One of the important correlations of HMBC was observed from the anomeric proton (H-1' to $\mathrm{C}-11)$, and the other correlation was observed from the anomeric proton $\left(\mathrm{H}-\mathrm{1}^{\prime}\right)$ to the position of glucose (C-6'). Indeed, acid hydrolysis of $\mathbf{4}$ afforded glucose and apiose. The specific rotations of glucose and apiose revealed 
that they were D-sugars $[23,24]$. The glycosyl groups were deduced from the ${ }^{13} \mathrm{C}-\mathrm{NMR}$ spectroscopic data and anomeric proton coupling constants as glucose and apiose having $\beta$-anomeric configuration $[25,26]$. Therefore, the new compound 4 was identified as 7-[( $\beta$-D-apiofuranosyl- $(1 \rightarrow 6)-$ $\beta$-D-glucopyranosyl)oxymethy]-8,8-dimethyl-4,8-dihydrobenzo[1,4]thiazine-3,5-dione.

\section{Experimental}

\subsection{General}

IR spectra were recorded on an IR-47 spectrometer. An optical-rotation detector (Shodex OR-2, Showa Denko Co., Ltd., Tokyo, Japan) was used. The optical rotation was recorded on a Perkin-Elmer 241 polarimeter. The melting points (uncorrected) were measured on a Kofler micromelting point apparatus. The HRESIMS analyses were conducted on an IonSpec Ultima 7.0T FTICR. The UV and NMR spectra were recorded on a Shimadzu UV-1601 and Bruker DPX 400 (400 MHz for ${ }^{1} \mathrm{H}-\mathrm{NMR}$ and $100 \mathrm{MHz}$ for ${ }^{13} \mathrm{C}$-NMR), respectively. Chemical shifts are given as $\delta$ values, with reference to that of tetramethylsilane (TMS), which was used as an internal standard. Coupling constants are given in Hz. Preparative HPLC (Waters, Delta 600-2487) was performed on a Hypersil-ODS II column (10 $\mu \mathrm{m}$, $20 \times 300$ mm, Yilite, Dalian, China). Semipreparative HPLC (Waters, Delta 600-2414-2998) was performed using a SunFire ${ }^{\mathrm{TM}}-\mathrm{C} 18$ column $(5 \mu \mathrm{m}, 10 \times 250 \mathrm{~mm}$, Waters, Milford, MA., USA). Silica gel (200-300 mesh, Yanghai, Qingdao, China) and ODS-A (120 A, $50 \mu \mathrm{m}$, YMC Co., Tokyo, Japan) were employed for column chromatography $(\mathrm{CC})$.

\subsection{Plant Material}

Fructus Xanthii was collected from Heilongjiang Province, China, in August 2011, and then authenticated by Prof. Wang Zhen-Yue, Heilongjiang University of Chinese Medicine, Harbin, China. A voucher specimen (No. 20111077) was deposited at the herbarium of Heilongjiang University of Chinese Medicine.

\subsection{Extraction and Isolation}

A $70 \%$ aqueous EtOH extract of Fructus Xanthii $(2.7 \mathrm{~kg})$ was partitioned between EtOAc and $\mathrm{H}_{2} \mathrm{O}$ and between $n$ - $\mathrm{BuOH}$ and $\mathrm{H}_{2} \mathrm{O}$. The $n-\mathrm{BuOH}$ extract $(59.4 \mathrm{~g})$ was subjected to silica gel chromatography using $\mathrm{CH}_{2} \mathrm{Cl}_{2}-\mathrm{MeOH}$ mixtures [20:1 (10 L), 10:1 (7 L), 5:1 (15 L), 2:1 (5 L) v/v] to afford fractions (Fr.) A-D. Fr.A was stored and Fr.B (12.1 g) was repeatedly subjected to CC on an ODS column using $\mathrm{MeOH}-\mathrm{H}_{2} \mathrm{O}$ solution [1:7 (3 L)-5:6 (4 L) v/v] as the mobile phase to afford compounds 1 (9 mg) and 6 (24 mg). Fr.C (20.3 g) was subjected to silica gel chromatography using $\mathrm{CH}_{2} \mathrm{Cl}_{2}-\mathrm{MeOH}$ mixtures [15:1 $(1.8 \mathrm{~L}), 5: 1(2.8 \mathrm{~L}) \mathrm{v} / \mathrm{v}$ ] to afford subfractions $\mathrm{C} 1(8.2 \mathrm{~g})$ and $\mathrm{C} 2$ $(9.7 \mathrm{~g})$. Fr.C2 was repeatedly subjected to separation using Sephadex LH-20 and $\mathrm{CH}_{2} \mathrm{Cl}_{2}-\mathrm{MeOH}$ solution $[1: 1(0.7 \mathrm{~L}) \mathrm{v} / \mathrm{v}]$ to yield Fr.C2-1 (4.6 g) and Fr.C2-2 (3.7 g). Fr.C2-2 was subjected to isocratic elution on an ODS column using $\mathrm{MeOH}-\mathrm{H}_{2} \mathrm{O}$ solution $[2: 1(0.5 \mathrm{~L}) \mathrm{v} / \mathrm{v}]$ to afford Fr.C2-2-1 $\left(1.0\right.$ g). Fr.C2-2-1 was finally purified by preparative HPLC using $\mathrm{MeOH}-\mathrm{H}_{2} \mathrm{O}$ solution (3:7, $\left.6 \mathrm{~mL} / \mathrm{min}, t_{\mathrm{R}}=8.5 \mathrm{~min} ; t_{\mathrm{R}}=9.5 \mathrm{~min}\right)$ to afford compounds $2(3.2 \mathrm{mg})$ and $\mathbf{3}(4.1 \mathrm{mg})$. Fr.D $(10.7 \mathrm{~g})$ was loaded on a Sephadex LH-20 column and eluted with $\mathrm{CH}_{2} \mathrm{Cl}_{2}-\mathrm{MeOH}$ solution [1:1 (0.8 L)] to 
yield five fractions, D1-D5. D2 (300 mg) was purified by semipreparative HPLC (isocratic elution) using $\mathrm{MeOH}-\mathrm{H}_{2} \mathrm{O}$ solution $\left(2: 3,3 \mathrm{~mL} / \mathrm{min}, \mathrm{t}_{\mathrm{R}}=12.7,18.0\right.$, and $\left.15.0 \mathrm{~min}\right)$ to afford compounds 4 (10.0 mg), 7 (19.0 mg), and $\mathbf{5}(57.1 \mathrm{mg})$.

3 $\beta$-Norpinan-2-one 3-O- $\beta$-D-apiofuranosyl- $(1 \rightarrow 6)$ - $\beta$-D-glucopyranoside $(\mathbf{1})$ : white amorphous powder $[\alpha]_{\mathrm{D}}^{20}-10.2$ (c 0.26, MeOH) UV $\lambda_{\max }(\mathrm{MeOH}) \mathrm{nm}(\log \varepsilon): 201(3.18) \mathrm{IR}(\mathrm{KBr}): 3560,3495,2980,1713$, 1075, $1042 \mathrm{~cm}^{-1}{ }^{1} \mathrm{H}-\mathrm{NMR}$ : Table $1{ }^{13} \mathrm{C}-\mathrm{NMR}$ : Table 2 HRESIMS $\mathrm{m} / \mathrm{z}[\mathrm{M}+\mathrm{Na}]^{+}$Calcd for $\mathrm{C}_{20} \mathrm{H}_{33} \mathrm{O}_{11} \mathrm{Na} 471.1842$, Found: $m / z 471.1847$.

(6Z)-3-Hydroxymethyl-7-methylocta-1,6-dien-3-ol 8-O- $\beta$-D-glucopyranoside (2): yellow amorphous powder $[\alpha]_{\mathrm{D}}^{20}-6.7$ (c 0.17, MeOH) UV $\lambda_{\max }(\mathrm{MeOH}) \mathrm{nm}(\log \varepsilon): 202(2.10) \mathrm{IR}(\mathrm{KBr}): 3378,2980,2830$, 1050, 917; $\mathrm{cm}^{-1}{ }^{1} \mathrm{H}-\mathrm{NMR}$ : Table $1{ }^{13} \mathrm{C}-\mathrm{NMR}$ : Table 2 HRESIMS $m / z[\mathrm{M}+\mathrm{Na}]^{+}$Calcd for $\mathrm{C}_{16} \mathrm{H}_{28} \mathrm{O}_{8} \mathrm{Na}$ 371.1682, Found: $m / z 371.1680$.

(6E)-3-Hydroxymethyl-7-methylocta-1,6-dien-3-ol 8-O- $\beta$-D-glucopyranoside (3): yellow amorphous powder $[\alpha]_{\mathrm{D}}^{20}-8.0(\mathrm{c} 0.19, \mathrm{MeOH}) \mathrm{UV} \lambda_{\max }(\mathrm{MeOH}) \mathrm{nm}(\log \varepsilon): 201(2.10) \mathrm{IR}(\mathrm{KBr}): 3380,2979,2833$, 1056, 919; $\mathrm{cm}^{-1}{ }^{1} \mathrm{H}-\mathrm{NMR}$ : Table $1{ }^{13} \mathrm{C}-\mathrm{NMR}$ : Table 2 HRESIMS $m / z[\mathrm{M}+\mathrm{Na}]^{+}$Calcd for $\mathrm{C}_{16} \mathrm{H}_{28} \mathrm{O}_{8} \mathrm{Na}$ 371.1682, Found: $m / z 371.1685$.

7-[( $\beta$-D-Apiofuranosyl-(1 $\rightarrow 6)$ - $\beta$-D-glucopyranosyl)oxymethy]-8,8-dimethyl-4,8-dihydrobenzo[1,4]thiazine3,5-dione (4): colorless needle crystals mp 190-192 ${ }^{\circ} \mathrm{C}[\alpha]_{\mathrm{D}}^{20}-10.7$ (c $\left.0.22, \mathrm{MeOH}\right) \mathrm{UV} \lambda_{\max }(\mathrm{MeOH})$ nm (log $\varepsilon$ ): 201(2.18), 246(2.87) IR (KBr): 3462, 1690; 1658, 1215, 1177, $929 \mathrm{~cm}^{-1}{ }^{1} \mathrm{H}-\mathrm{NMR}$ : Table 1 ${ }^{13} \mathrm{C}-\mathrm{NMR}$ : Table 2 HRESIMS $m / z[\mathrm{M}+\mathrm{H}]{ }^{+}$Calcd for $\mathrm{C}_{22} \mathrm{H}_{32} \mathrm{NO}_{12} \mathrm{~S} 534.1645$, Found: $m / z$ 534.1650.

\subsection{Acid hydrolysis of $\mathbf{1}, \mathbf{2}, \mathbf{3}$, and $\mathbf{4}$}

Compound $1(3.0 \mathrm{mg})$ was dissolved in $2 \mathrm{M} \mathrm{HCl}(2.0 \mathrm{~mL})$ and then heated at $80{ }^{\circ} \mathrm{C}$ in a water bath for $2 \mathrm{~h}$. The reaction mixture was diluted with $\mathrm{H}_{2} \mathrm{O}(20 \mathrm{~mL})$ and then extracted with $n$-BuOH $(20 \mathrm{~mL})$. The aqueous layer was neutralized with Amberlite MB-3 (Organo Co., Ltd., Tokyo, Japan), and then dried under reduced pressure to afford the monosaccharide fraction. The monosaccharide fraction was extracted with $\mathrm{MeOH}$, and the $\mathrm{MeOH}$ extract was analyzed using HPLC under the following conditions: HPLC column, Kaseisorb LC NH$-60-5$ (4.6 mm i.d. 250 mm; Tokyo Kasei Co., Ltd., Tokyo, Japan); detection, optical rotation (Shodex OR-2, Showa Denko Co., Ltd., Tokyo, Japan); mobile phase, $\mathrm{CH}_{3} \mathrm{CN}-\mathrm{H}_{2} \mathrm{O}(17: 3, \mathrm{v} / \mathrm{v})$; flow rate, $1.0 \mathrm{~mL} / \mathrm{min}$. Identification of D-glucose and $\mathrm{D}$ apiose from 1 was carried out by comparing their retention times and optical rotations with those of authentic samples. The values of $t_{\mathrm{R}}$ were $11.5 \mathrm{~min}$ (D-glucose, positive optical rotation) and $5.8 \mathrm{~min}$ (D-apiose, positive optical rotation). Through the same method, the monosaccharides were identified as D-glucose for $\mathbf{2}$ and 3, and D-glucose and D-apiose for $\mathbf{4}$.

\section{Conclusions}

As a part of our chemical investigation on Fructus Xanthii, four new glycosides, namely $3 \beta$-norpinan-2-one 3-O- $\beta$-D-apiofuranosyl-( $1 \rightarrow 6)$ - $\beta$-D-glucopyranoside $(\mathbf{1})$, (6Z)-3-hydroxymethyl-7methylocta-1,6-dien-3-ol 8-O- $\beta$-D-glucopyranoside (2), (6E)-3-hydroxymethyl-7-methylocta-1,6-dien- 
3-ol 8-O- $\beta$-D-glucopyranoside (3), and 7-[( $\beta$-D-apiofuranosyl-( $1 \rightarrow 6)-\beta$-D-glucopyranosyl)oxymethy]8,8-dimethyl-4,8-dihydrobenzo[1,4]thiazine-3,5-dione (4) were isolated. Their structures were established on the basis of spectroscopic evidence. This discovery represents a further addition to the number and diversity of glycosidic compounds.

\section{Acknowledgments}

We thank Wei Guo Zhu for the NMR measurements at Zhengzhou University. We are also grateful to the Key Laboratory of Chinese Materia Medica, Ministry of Education.

\section{Conflicts of Interest}

The authors declare no conflict of interest.

\section{References}

1. The State of Pharmacopoeia Commission of People's Republic of China. Pharmacopoeia of People's Republic of China; Chemical Industry Press: Beijing, China, 2010; p. 151.

2. Jiangsu New Medical College. Dictionary of Chinese Materia Medica; Shanghai Scientific Technological Publishers: Shanghai, China, 1985; pp. 1071-1072.

3. Kinghorn, A.D.; Farnsworth, N.R.; Soejarto, D.D.; Cordell, G.A.; Pezutto, J.M.; Udeani, G.O.; Wani, M.C.; Wall, M.E.; Navarro, H.A.; Kramer, R.A.; et al. Novel strategies for the discovery of plant-derived anti-cancer agents. Pure Appl. Chem. 1999, 71, 1611-1618.

4. Sato, Y.; Oketani, H.; Yamada, T.; Singyouchi, K.; Ohtsubo, T.; Kihara, M.; Shibata, H.; Hi, T. A xanthanolide with potent anti-bacterial activity against methicillin-resistant Staphylococcus aureus. J. Pharm. Pharmacol. 1997, 49, 1042-1044.

5. Saxena, V.K.; Mondal, S.K. A xanthanolide from Xanthium strumarium. Phytochemistry 1994, $35,1080-1082$.

6. Turgut, M.; Alhan, C.C.; Gürgöze, M.K.A.; Doğan, Y.; Tekatli, M.; Akpolat, N.; Aygün, A.D. Carboxyatractyloside poisoning in humans. Ann. Trop. Paediatr. 2005, 25, 125-134.

7. West, P.L.; McKeown, N.J.; Hendrickson, R.G. Muscle spasm associated with therapeutic use of Cang Er Zi Wan. Clin. Toxicol. (Phila.) 2010, 48, 380-384.

8. Chen, B.; Ma, L.; Wang, X.; Shen, Y.; Jia, X. Simultaneous determination of 5 phenolic acids in fried Fructus xanthii from different production sites and its dispensing granules by using ultra-pressure liquid chromatography. Pharmacogn. Mag. 2013, 9, 103-108.

9. Kan, S.; Chen, G.; Han, C.; Chen, Z.; Song, X.; Ren, M.; Jiang, H. Chemical constituents from the roots of Xanthium sibiricum. Nat. Prod. Res. 2011, 25, 1243-1249.

10. Wang, L.; Wang, J.; Li, F.; Liu, X.; Chen, B.; Tang, Y.X.; Wang, M.K. Cytotoxic sesquiterpene lactones from aerial parts of Xanthium sibiricum. Planta Med. 2013, 79, 661-665.

11. Zhang, L.; Tao, L.; Ruan, J.; Li, W.; Wu, Y.; Yan, L.; Zhang, F.; Fan, F.; Zheng, S.; Wang, A.; $\mathrm{Lu}, \mathrm{Y}$. Xanthatin induces $\mathrm{G} 2 / \mathrm{M}$ cell cycle arrest and apoptosis in human gastric carcinoma MKN-45 cells. Planta Med. 2012, 78, 890-895. 
12. Ma, Y.T.; Huang, M.C.; Hsu, F.L.; Chang, H.F. Thiazinedione from xanthium strumarium. Phytochemistry 1998, 48, 1083-1085.

13. Roussakis, C.; Chinou, I.; Vayas, C.; Harvala, C.; Verbist, J.F. Cytotoxic activity of xanthatin and the crude extracts of Xanthium strumarium. Planta Med. 1994, 60, 473-474.

14. Dai, Y.H.; Cui, Z.; Li, J.L.; Wang, D. A new thiaziedione from the fruits of Xanthium sibiricum. J. Asian Nat. Prod. Res. 2008, 10, 343-347.

15. Ahuja, M.; Nigam, S. Chemical examination of essential oil from the leaves of Xanthium strumarium (Linn.). Flavour Ind. 1970, 1, 627-630.

16. Taher, H.A.; Ubiergo, G.O.; Talenti, E.C.J. Constituents of the essential of Xanthium cavanillesii. J. Nat. Prod. 1985, 48, 857.

17. MacLeod, J.K.; Moeller, P.D.; Franke, F.P. Two toxic kaurene glycosides from the burrs of Xanthium pungens. J. Nat. Prod. 1990, 53, 451-455.

18. Han, T.; Li, H.L.; Zhang, Q.Y.; Han, P.; Zheng, H.C.; Rahman, K.; Qin, L.P. Bioactivity-guided fractionation for anti-inflammatory and analgesic properties and constituents of Xanthium strumarium L. Phytomedicine 2007, 14, 825-829.

19. An, H.J.; Jeong, H.J.; Lee, E.H.; Kim, Y.K.; Hwang, W.J.; Yoo, S.J.; Hong, S.H.; Kim, H.M. Xanthii fructus inhibits inflammatory responses in LPS-stimulated mouse peritoneal macrophages. Inflammation 2004, 28, 263-270.

20. Zheng, X.K.; Bi, Y.F.; Feng, W.S. Study on the chemical constituents of Selaginellatamariscina (Beauv.) Spring. Acta Pharm. Sin. 2004, 39, 266-268.

21. Beate, B.; Peter, W. Isolation and characterization of novel benzoates, cinnamates, flavonoids, and lignans from Riesling wine and screening for antioxidant activity. J. Agric. Food Chem. 2001, 49, 2788-2798.

22. Pierre, L.; Gilles, B. Efficient conversion of (1R,5R)-(+)- $\alpha$-pinene to (1S,5R)-(-)-nopinone. J. Org. Chem. 1986, 51, 1362-1365.

23. Masateru, O.; Fumie, S.; Syoko, S.; Chika, T.; Hitoshi, Y. Three new steroid glycosides from the underground parts of Trillium kamtschaticum. Chem. Pharm. Bull. 2007, 55, 1093-1096.

24. Qiu, Y.K.; Chen, Y.J.; Pei, Y.P. Constituents with radical scavenging effect from Opuntiadillenii: Structures of new $\alpha$-pyrones and flavonol glycoside. Chem. Pharm. Bull. 2002, 50, 1507-1510.

25. Wang, Z.B.; Gao, H.Y.; Xu, F.M.; Wu, L.J. Three new compounds from the leaves of Acanthopanaxseticosus Harms. Chin. Chem. Lett. 2010, 21, 702-705.

26. Isao, K.; Masahiro, S.; Fumi, H.; Zhou, J.L.; My, R.J.L. Apioglycyrrhizin and araboglycyrrhizin, two new sweet oleanene-type triterpeneoligoglycosides from the root of glycyrrhizainflata. Chem. Pharm. Bull. 1989, 37, 551-553.

27. Nakamura, S.; Li, X.Z.; Matsuda, H.; Yoshikawa, M. Bioactive constituents from Chinese natural medicines. XXVIII. ${ }^{1)}$ Chemical structures of acyclic alcohol glycosides from the roots of Rhodiola crenulata. Chem. Pharm. Bull. 2008, 56, 536-540.

Sample Availability: Samples of the compounds 1-4 are available from the authors.

(C) 2013 by the authors; licensee MDPI, Basel, Switzerland. This article is an open access article distributed under the terms and conditions of the Creative Commons Attribution license (http://creativecommons.org/licenses/by/3.0/). 